\title{
Magnetic resonance susceptibility weighted in evaluation of cerebrovascular diseases
}

\author{
Amr Yehia Abd Elmotelb Sultan, Khalid Ismael ElShafey, Omar Ahmed Hassanien and Rasha Mahmoud Dawoud ${ }^{*}$
}

\begin{abstract}
Background: Cerebrovascular diseases are considered a very hard burden as they may lead to poor outcome, and they are considered the second most common cause of morbidity and mortality after coronary artery disease. They include wide variety of diseases that affect vascularity of brain tissue with the most common one is stroke-either ischemic or hemorrhagic. The aim of the current study was to assess the role of susceptibility weighted imaging (SWI) in imaging of different cerebrovascular diseases and what would be added by SWI to different routine magnetic resonance imaging (MRI) sequences.

Results: Fifty-five patients enrolled in this study, 14 patients had lesions with calcifications, 13 patients had cavernoma, 10 patients had diffuse axonal injury, 11 patients with infarction, 2 patients with AVM, 2 patients with chronic microbleed, 2 patients with hemorrhage, and 1 patient with hemorrhagic tumor, and the result showed that SWI has sensitivity $100 \%$, specificity $60 \%$, and accuracy $91.9 \%$ in regard to diagnosis of cavernoma while sensitivity $91.7 \%$, specificity $50 \%$, and accuracy $85.7 \%$ in regard to diagnosis of calcification and regarding diagnosis of diffuse axonal injury; SWI has $98.3 \%$ sensitivity, $100 \%$ specificity, and $98.4 \%$ accuracy; finally, in regard to diagnosis of hemorrhagic lesions, SWI has $96.1 \%$ sensitivity, $66.7 \%$ specificity, and $93.1 \%$ accuracy.

Conclusion: SWI is very sensitive in the diagnosis and detection of actual number of vascular malformation like cavernomas than conventional MRI. SWI adds significant diagnostic value to routine MRI sequences in regard to calcification that was nearly limited in its diagnosis by $\mathrm{CT}$. Diagnosis of microbleeds becomes easier and accurate with SWI. Diffuse axonal injury was and still considered a clinical diagnosis, but SWI becomes the gold standard in its imaging diagnosis confirming the clinical one.
\end{abstract}

Keywords: Susceptibility weighted imaging, Cerebrovascular diseases, Vascular malformation

\section{Background}

Cerebrovascular disease includes a variety of medical conditions that affect the blood vessels of the brain and the cerebral circulation. It is considered the second leading cause of morbidity and mortality after coronary artery diseases. There are many causes of cerebrovascular diseases like traumatic brain injury and stroke which are the most common and the medical emergency that requires prompt treatment to minimize brain damage and potential complications, and less common and less severe diseases like cavernous malformation, arteriovenous fistula, amyloid

\footnotetext{
* Correspondence: roshy_dawoud@yahoo.com

Radiodiagnosis \& Medical Imaging Department, Faculty of Medicine, Tanta University, El-geish street, Tanta, Gharbya Governorate, Egypt
}

angiopathy, developmental venous anomaly, etc., that are usually asymptomatic until an acute event such as a stroke occurs. The diagnosis of cerebrovascular diseases is clinical with assistance from imaging techniques like computed tomography (CT) scan and magnetic resonance imaging (MRI) [1-3].

MRI uses different sequences for imaging like T1weighted images (T1W), T2-weighted images (T2W), diffusion weighted images (DWI), proton density images (PDI), and fluid attenuation inversion recovery (FLAIR) in addition to new sequences exploiting higher field strength like arterial spin labelling (ASL) and susceptibility weighted imaging (SWI) $[3,4]$.
Springer Open (c) The Author(s). 2020 Open Access This article is licensed under a Creative Commons Attribution 4.0 International License which permits use, sharing, adaptation, distribution and reproduction in any medium or format, as long as you give appropriate credit to the original author(s) and the source, provide a link to the Creative Commons licence, and indicate if changes were made. The images or other third party material in this article are included in the article's Creative Commons licence, unless indicated otherwise in a credit line to the material. If material is not included in the article's Creative Commons licence and your intended use is not permitted by statutory regulation or exceeds the permitted use, you will need to obtain permission directly from the copyright holder. To view a copy of this licence, visit http://creativecommons.org/licenses/by/4.0/. 
Susceptibility weighted imaging (SWI) is one of the most important of these sequences. It is a fully flow compensated, three-dimensional, high spatial resolution, and gradient echo MR imaging technique. The basic concept of this technique is maintaining phase information into the final image, discarding phase artifacts, and keeping just the local phase of interest. Thus, it utilizes the phase information and signal loss to reveal the susceptibility differences between various tissues and substances like blood products, iron, and calcification $[5,6]$.

SWI is used as a complimentary tool to conventional MR imaging sequences, and its importance has been demonstrated in various neurological disorders as it is sensitive to both diamagnetic and paramagnetic substances like deoxygenated blood and intracranial mineral deposition which generate different phase shift in MRI data. SWI images can be displayed as a minimum intensity projection that provides high resolution delineation of the cerebral venous architecture, a feature that is not available in other MRI techniques [7-9].

The aim of the current study was to assess the role of SWI in imaging of different cerebrovascular diseases and what would be added by SWI to different routine MRI sequences in the form of comparison between them by various statistical tests like chi-square test, sensitivity, specificity, and accuracy and also by the assessment of the detection rate to finally evaluate some of the clinical applications of SWI.

\section{Methods}

\section{Study design and population}

This prospective study was carried on 55 patients (32 males, 23 females) with their age ranged from 1 year and 4 months to 64 years old, at the period between June 2018 and June 2019; the patients had wide variety of cerebrovascular diseases and were examined by conventional MRI sequences and SWI. CT was done in some patients especially with calcification.

Ethics committee approved, and informed consent were obtained for all patients or their guardians. Privacy and confidentiality of all patients' data were guaranteed; all data provision were monitored and used for security purpose only.

\section{Inclusion criteria}

A. Patients with suspected symptoms of stroke or vascular malformations are as follows:

- Confusion, including trouble with speaking and understanding.

- Headache, possibly with altered consciousness or vomiting.
- Numbness or inability to move parts of the face, arm, or leg, particularly on one side of the body.

- Walking troubles, dizziness, and lack of coordination.

- Vision loss.

- Severe unsteadiness.

B. Patients presented with trauma and non-contrast CT were free or revealed few small hyperdense foci.

C. Patients with past history of brain tumor are presented with new symptoms suspecting complications like hemorrhagic transformation.

\section{Exclusion criteria}

A. Patients with metallic medical implants (intraocular metallic foreign body, cardiac pace makers, MR non compatible intracranial clips of arterial brain aneurysms).

B. Unfit patients for examination.

- Patient who presented with impaired cognition and uncontrolled movement.

- Patients with some mental disorders.

C. Patients who refused to do the examination.

\section{Preparation and protocol}

All patients in this study were submitted to complete history taking, clinical examination (by neuropsychiatry physicians), and patient preparation including removing of any detachable metallic implants in patient body or clothes like keys and teeth prosthesis prior to entrance to magnetic area.

The patients were placed in the supine position and the head in the neutral position with the use of a circular polarized head-array coil and ultra gradients. All MRI scans were performed by a MRI $1.5 \mathrm{~T}$ unit ( GE Signa Explorer) closed magnet right-handed system using a head coil in radiodiagnosis and medical imaging department, with magnetic resonance imaging protocol included Axial T1WI (TR/TE $=400-600 / 10-20 \mathrm{~m} / \mathrm{s}$ and FOV $=180 \mathrm{~mm})$, Axial T2WI $(\mathrm{TR} / \mathrm{TE}=2000-4000 /$ $100-120 \mathrm{~m} / \mathrm{s}$, and FOV $=180 \mathrm{~mm}$ ), coronal and/or axial FLAIR images (TR/TE/inversion time $(\mathrm{TI})=4000-$ $6000 / 140 / 1200$ and FOV $=180 \mathrm{~mm}$ ), and diffusion weighted imaging ) using $b$ value of 0 and 1000) with apparent diffusion coefficient calculation that were obtained by using an axial echo-planar SE sequence and susceptibility weighted imaging (SWI). 


\section{Susceptibility weighted imaging (SWI)}

Susceptibility weighted images were generated from gradient-echo (GRE) pulse sequences with typical imaging parameters $(\mathrm{TR} / \mathrm{TE}=6400 / 30 \mathrm{~ms}, \mathrm{FOV}=280$ $\mathrm{mm}$, acquisition matrix $=256 \times 256$, slice thickness $=$ $0.2 \mathrm{~mm}$, gap $=2 \mathrm{~mm}$, and scan time $=6 \mathrm{~min}$ and $10 \mathrm{~s}$ with MIP reconstruction for the image).

A key feature of SWI was that magnitude and phase information were independently processed/displayed as well as combined for diagnostic purposes.

The magnitude image was saved for diagnostic purposes, displaying background tissue with spin-densitylike contrast while the raw phase image was dominated by large but slowly changing "macro"-susceptibility gradients due to generalized field in homogeneities as well as distortions due to air and bone at the skull base. Digital high-pass filtering could be used to remove these low-frequency fluctuations, and additional local phase correction algorithms may be employed to reduce artifacts at the skull base. The end result is a filtered phase image that was saved and used for diagnostic purposes.

A phase mask was next created that scales data from the filtered phase images over a $0-1$ range to accentuate tissues with different susceptibilities. The magnitude image was digitally multiplied by this phase mask several times until the desired mix of phase information was imparted. The end result was a susceptibility weighted (SW) image, which simultaneously contained both magnitude and phase information and was used for clinical diagnosis. Minimum intensity projections (mIP) of the SW image were typically displayed, which vary in thickness depending on how many of the original 3D source images were stacked together.

A comparison between the SW image and the filter phase was made for interpretation, but firstly we make sure that venous structures display hypointense signal in filter phase, if it is hyperintense, then reverse the window first for correct interpretation.

Both bleeding and calcification displayed hypointense signal in SW image and would be differentiated on the filter phase. Bleeding displayed hypointense signal in filter phase, and calcification displayed hyperintense signal in filter phase; these results were reversed in left handed MR system.

The interpretation of the images was done by two expert radiologist who had experience in neuroradiology 18 and 10 years and in functional radiology for more than 6 years .All cases reviewed blindly, and the decision was taken together.

\section{Statistical analysis}

All the data were entered into Excel sheet Microsoft Office Excel 2007 and analyzed statistically using the SPSS Statistical software (version 20.0.0: IBM Corp: Armonk, NY) and XL-STAT (Addinsoft, NY, USA).
- All the outcome variables, i.e., quantitative data, were summarized in the form of mean \pm standard deviation with the used tests were chi-square test for categorical variables, to compare between different groups, sensitivity that is the capacity of the test to correctly identify diseased individuals in a population "TRUE POSITIVES." The greater the sensitivity, the smaller the number of unidentified case "false negatives," specificity that is the capacity of the test to correctly exclude individuals who are free of the disease "TRUE NEGATIVES." The greater the specificity, the fewer "false positives" will be included, accuracy (rate of agreement $=($ true positives + true negatives $) /$ total tested $\times 100)$ and probability ( $P$ value): $P$ value $<0.05$ was considered significant. $P$ value $>0.05$ was considered insignificant.

\section{Results}

This study included 55 patients; the age of the patients ranged from 1 year and 4 months to 64 years old with a mean of $34 \pm 14.9$ years and median of 43.5 years; fourteen patients $(25.4 \%)$ had calcifications at different sites with different pathologies (either in calcified oligodendroglioma, teratoma, basal ganglia calcification, calcified granuloma or sporadically), 13 patients (23.6\%) had cavernomas, 11 patients $(20 \%)$ had infarction (9 patients had hemorrhagic infarction and 2 patients had nonhemorrhagic infarction), 10 patients (18.1\%) had diffuse axonal injury (DAI), 2 patients (3.6\%) had AVM, 2 patients (3.6\%) had microbleeds, 2 patients (3.6\%) had hemorrhage, and 1 patient (1.8\%) had hemorrhagic tumor (Table 1$)$.

\section{Regarding the diagnosis of cavernoma (Fig. 1)}

Among the 55 patients examined in this study, 13 patients were diagnosed with cavernoma(either sporadic or inherited) on the basis of specific signals on different MRI sequences with no obvious common risk factor between the patients, but with positive family history of the same pathology in 9 patients. As cavernoma is commonly being multiple in number especially in inherited type so in the current study, the total number of cavernomas detected in 13 patients was 33 lesions.

The Pearson chi-square value for SWI in comparison to conventional MRI sequences (T1WI + T2WI) was 34.803 , and the $P$ value was 0.004 while the chi-square value for T1WI + T2WI in comparison to SWI was 47.95 , and the $P$ value was 0.086 .

Regarding to the final outcome of the reviewed 33 cavernoma lesions depending on the comparison with other conventional MRI sequences and CT, SWI sequence defined 30 lesions (90.9\%) as positive and 3 lesions $(9.1 \%)$ as negative. There were 28 true positive, 2 false positive which were misdiagnosed and confirmed to be calcification by CT, 3 true negative, and no false negative studies. On the other hand, T1WI defined 14 
Table 1 Types of pathological lesions included in the current study

\begin{tabular}{|c|c|c|c|c|}
\hline \multirow{2}{*}{$\frac{\text { Pathological lesion }}{\text { Calcification }}$} & \multicolumn{2}{|c|}{ Number of patients } & \multirow{2}{*}{$\begin{array}{l}\text { Types of lesions } \\
\text { Calcified oligodendroglioma }\end{array}$} & \multirow{2}{*}{$\frac{\text { Percentage }(\%)}{25.4}$} \\
\hline & 14 & 2 & & \\
\hline & & 2 & Teratoma & \\
\hline & & 3 & Basal ganglia calcification & \\
\hline & & 1 & Calcified granuloma & \\
\hline & & 6 & Sporadic calcification & \\
\hline Cavernoma & 13 & & & 23.6 \\
\hline \multirow[t]{2}{*}{ Infarction } & 11 & 9 & Hemorrhagic infarction & 20 \\
\hline & & 2 & Non-hemorrhagic infarction & \\
\hline Diffuse axonal injury & 10 & & & 18.1 \\
\hline AVM & 2 & & & 3.6 \\
\hline Chronic microbleeds & 2 & & & 3.6 \\
\hline Hemorrhage & 2 & & & 3.6 \\
\hline Hemorrhagic tumor & 1 & & & 1.8 \\
\hline
\end{tabular}

(42.42\%) lesions as positive and 19 (57.57\%) as negative. There were 12 true positive, 2 false positive, 1 true negative, and 18 false negative studies; also, T2WI defined 21 (63.63\%) lesions as positive and $12(36.36 \%)$ as negative. There were 19 true positive, 2 false positive, 1 true negative, and 11 false negative studies with sensitivity, specificity, and accuracy as shown in Table 2.

\section{Regarding the detection of calcification (Fig. 2)}

Among the 55 patients examined in this study, 14 patients were diagnosed with different pathologies containing calcification, either sporadically or in calcified tumor like teratoma, oligodendroglioma and meningioma, or calcified granuloma; conventional MRI cannot accurately diagnose calcification, and there are usually diverse signals or no signal at calcification sites in T1WI and T2WI. In this study, just detection of altered signal intensity in conventional MRI regarding calcification sites was done and compared with SWI and computed tomography (CT) taken as the control.

The Pearson chi-square value for SWI in comparison to T1WI was 42.507 , and the $P$ value was 0.032 . So SWI performed significantly better in detection of calcification lesions than T1WI, and the Pearson chi-square value for SWI in comparison to T2WI was 47.2, and the $P$ value was 0.078 which is statistically insignificant probably due to decreased number of tested patients.

Regarding the final outcome of the reviewed 14 calcification lesions depending on the comparison with $\mathrm{CT}$, SWI sequence defined 12 lesions (85.7\%) as positive and 2 lesions (14.2\%) as negative. There were 11 true positive, 1 false positive which were misdiagnosed and confirmed not to be calcification by CT, 1 true negative, and 1 false negative study with sensitivity, specificity, and accuracy as shown in Table 3.
Regarding the diagnosis of diffuse axonal injury (DAI) (Fig. 3)

Among the 55 patients examined in this study, 10 patients were diagnosed with DAI with its different grading based on location of scattered microbleeds. The total number of microbleeds in the 10 patients was 62 lesions detected.

The Pearson chi-square value for SWI in comparison to conventional MRI sequences (T1WI + T2WI) was 37.8 , and the $P$ value was 0.0067 while the chi-square value for (T1WI + T2WI) in comparison with SWI was 52.06 , and the $P$ value was 0.2 . So SWI performed significantly better in detecting DAI lesions (microbleeds) than conventional MRI.

Regarding the final outcome of the reviewed 62 DAI lesions (microbleeds) depending on the comparison with other conventional MRI sequences and CT, SWI sequence defined 59 lesions (95.2\%) as positive and 3 lesions $(4.8 \%)$ as negative. There were 59 true positive, no false positive studies, 2 (3.2\%) true negative, and 1 (1.6\%) false negative studies. On the other hand, T1WI defined 36 lesions (58\%) as positive and 26 lesions (41.9\%) as negative. There were $34(54.9 \%)$ true positive, $2(3.2 \%)$ false positive, $2(3.1 \%)$ true negative, and 24 (38.7\%) false negative studies; also, T2WI defined 32 lesions (51.6\%) as positive and $30(48.3 \%)$ as negative. There were 30 $(48.3 \%)$ true positive, $2(3.1 \%)$ false positive, $2(3.1 \%)$ true negative, and 28 (45.1\%) false negative studies with sensitivity, specificity, and accuracy as shown in Table 4.

\section{Regarding the detection of hemorrhage (Figs. 4 and 5)}

In the current study, 16 patients diagnosed with hemorrhage due to several causes like bleeding AVM, hemorrhagic tumor, hemorrhagic infarction, chronic microbleeds, and 

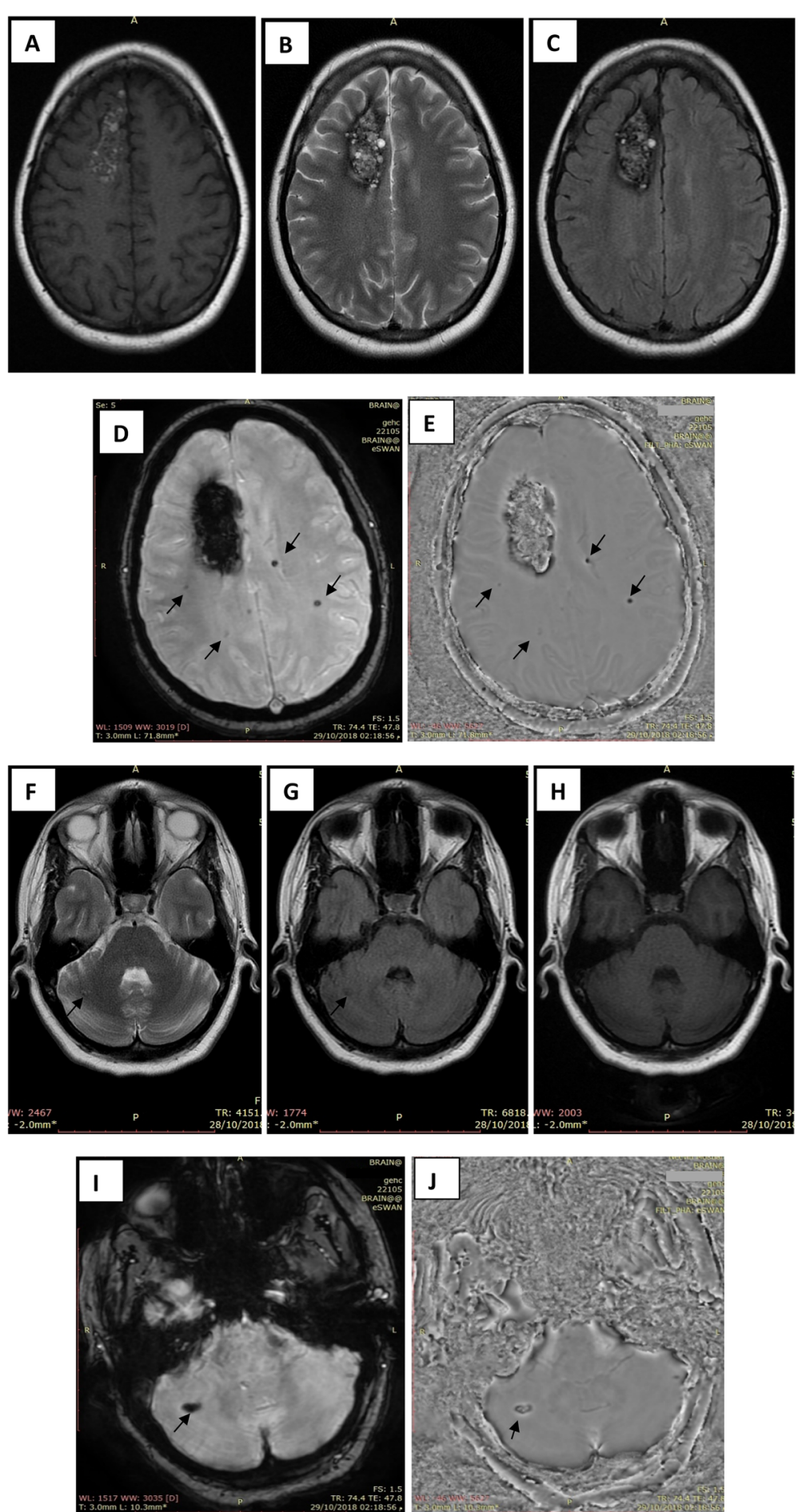

Fig. 1 Female patient aged 45 years old presented with severe headache and confusion. MRI brain was done and showed right upper frontal cavernoma together with multiple microbleeds that were inconspicuous in T1WI, T2WI, and FLAIR sequences (a, b, and $\mathbf{c}$ ) but are evident in SWI (d) confirmed by low signal intensity in filter phase (e). Lower level in the same patient showed another right much smaller cerebellar cavernoma that was overlooked by conventional MRI sequences, faint hyperintense in T2WI (f), and faint hypointense in FLAIR (g), and no lesion could be detected in T1 WI (h) but becomes obvious in SWI and filter phase (i and $\mathbf{j}$ ) 
Table $2 \mathrm{~T} 1 \mathrm{WI}, \mathrm{T} 2 \mathrm{Wl}$, and SWI lesion signal intensity in the cavernoma (number of patients = 13 and number of lesions = 33)

\begin{tabular}{|c|c|c|c|c|c|c|}
\hline \multirow{2}{*}{$\begin{array}{l}\text { MRI } \\
\text { sequence }\end{array}$} & \multicolumn{3}{|c|}{ Number of lesions } & \multirow{2}{*}{$\begin{array}{l}\text { Sensitivity } \\
(\%)\end{array}$} & \multirow{2}{*}{$\begin{array}{l}\text { Specificity } \\
(\%)\end{array}$} & \multirow{2}{*}{$\begin{array}{l}\text { Accuracy } \\
(\%)\end{array}$} \\
\hline & Isointense & Hypointense & Hyperintense & & & \\
\hline T1WI & 19 & 0 & 14 & 40 & 33.3 & 39.4 \\
\hline T2WI & 12 & 0 & 21 & 63.3 & 33.3 & 60.6 \\
\hline SWI & 3 & 30 & 0 & 100 & 60 & 93.9 \\
\hline$P$ value & \multicolumn{6}{|c|}{$0.004^{*}$ for SWI and $0.086^{*}$ for $(\mathrm{T} 1 \mathrm{WI}+\mathrm{T} 2 \mathrm{WI})$} \\
\hline
\end{tabular}

${ }^{*}$ Chi-square test, $P$ value $<0.05$ is considered statistically significant

intraparenchymal hemorrhage were examined with routine MRI sequences and SWI.

The Pearson chi-square value for SWI in comparison to conventional MRI sequences (T1WI + T2WI) was 43.06, and the $P$ value was 0.028 while the chi-square value for (T1WI + T2WI) in comparison to SWI was
48.03, and the $P$ value was 0.094 . So SWI performed significantly better in detecting hemorrhagic lesions than conventional MRI.

Regarding to the final outcome of the reviewed 29 hemorrhagic lesions depending on the comparison with other conventional MRI sequences and CT, SWI
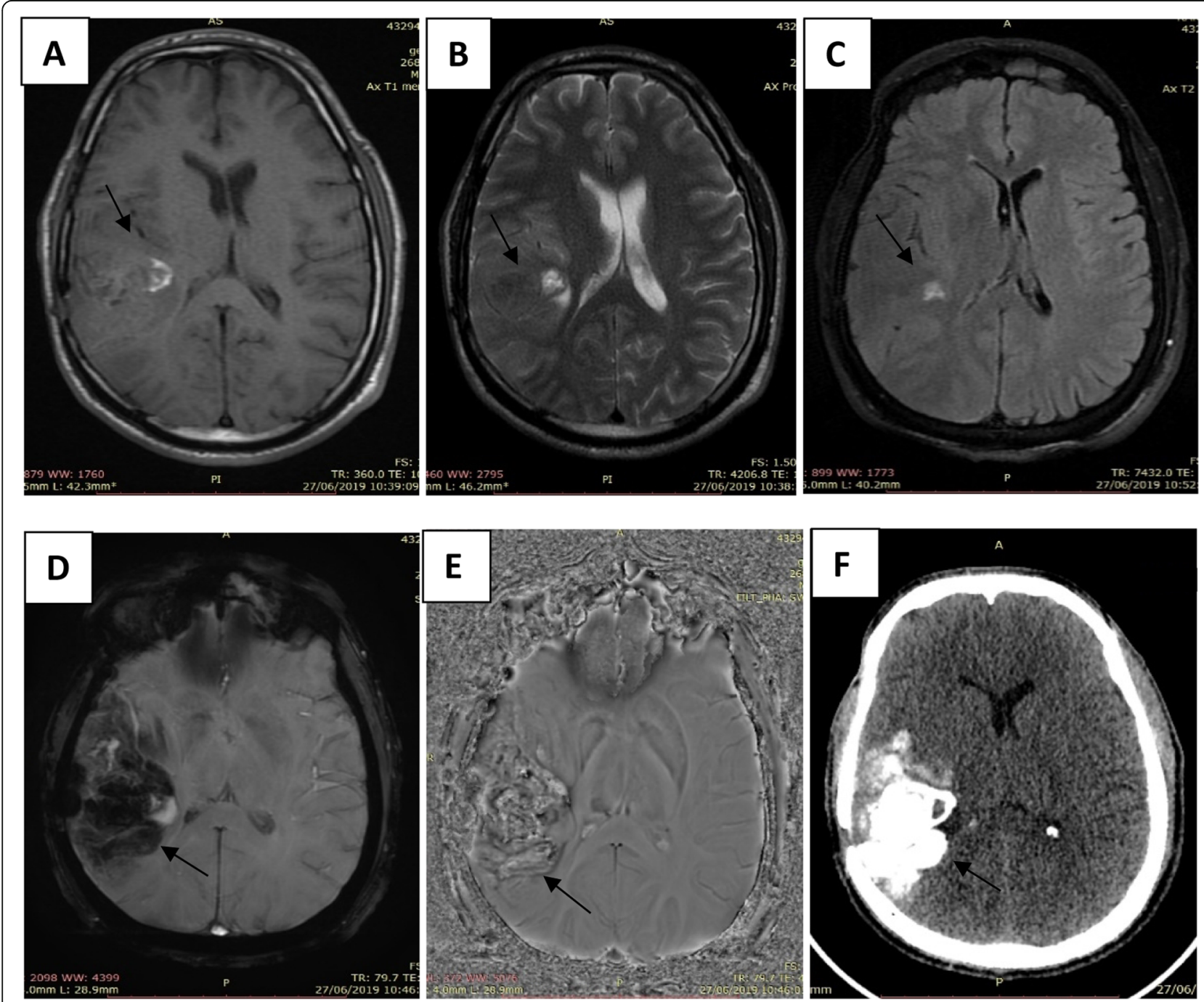

Fig. 2 Male patient aged 39 years old, presented with repeated headache and drowsiness with past history of oligodendroglioma that was surgically removed. Current MRI showed right tempro-parietal space occupying lesion with non-specific signal intensity in T1WI, T2WI, and FLAIR sequences $(\mathbf{a}, \mathbf{b}$, and $\mathbf{c}$ ) showing large intervening low signal intensity in SWI $(\mathbf{d})$, high signal intensity in filter phase (e), and corresponding large hyperdense area in $\mathrm{CT}(\mathbf{f})$ denoting calcification mostly recurrent calcified oligodendroglioma 
Table $3 \mathrm{~T} 1 \mathrm{WI}, \mathrm{T} 2 \mathrm{WI}$, and SWI lesion signal intensity of the calcification (number of patients = 14)

\begin{tabular}{|c|c|c|c|c|c|c|}
\hline \multirow{2}{*}{$\begin{array}{l}\text { MRI } \\
\text { sequence }\end{array}$} & \multicolumn{3}{|c|}{ Number of lesions } & \multirow{2}{*}{$\begin{array}{l}\text { Sensitivity } \\
(\%)\end{array}$} & \multirow{2}{*}{$\begin{array}{l}\text { Specificity } \\
(\%)\end{array}$} & \multirow{2}{*}{$\begin{array}{l}\text { Accuracy } \\
(\%)\end{array}$} \\
\hline & Isointense & Hypointense & Hyperintense & & & \\
\hline T1WI & 7 & 3 & 4 & & & \\
\hline $\mathrm{T} 2 \mathrm{WI}$ & 5 & 4 & 5 & & & \\
\hline SWI & 2 & 12 & 0 & 91.7 & 50 & 85.7 \\
\hline$P$ value & \multicolumn{6}{|c|}{$0.032^{*}$ for SWI with T1WI and $0.078^{*}$ for SWI with T2WI } \\
\hline
\end{tabular}

${ }^{*}$ Chi-square test, $P$ value $<0.05$ is considered statistically significant
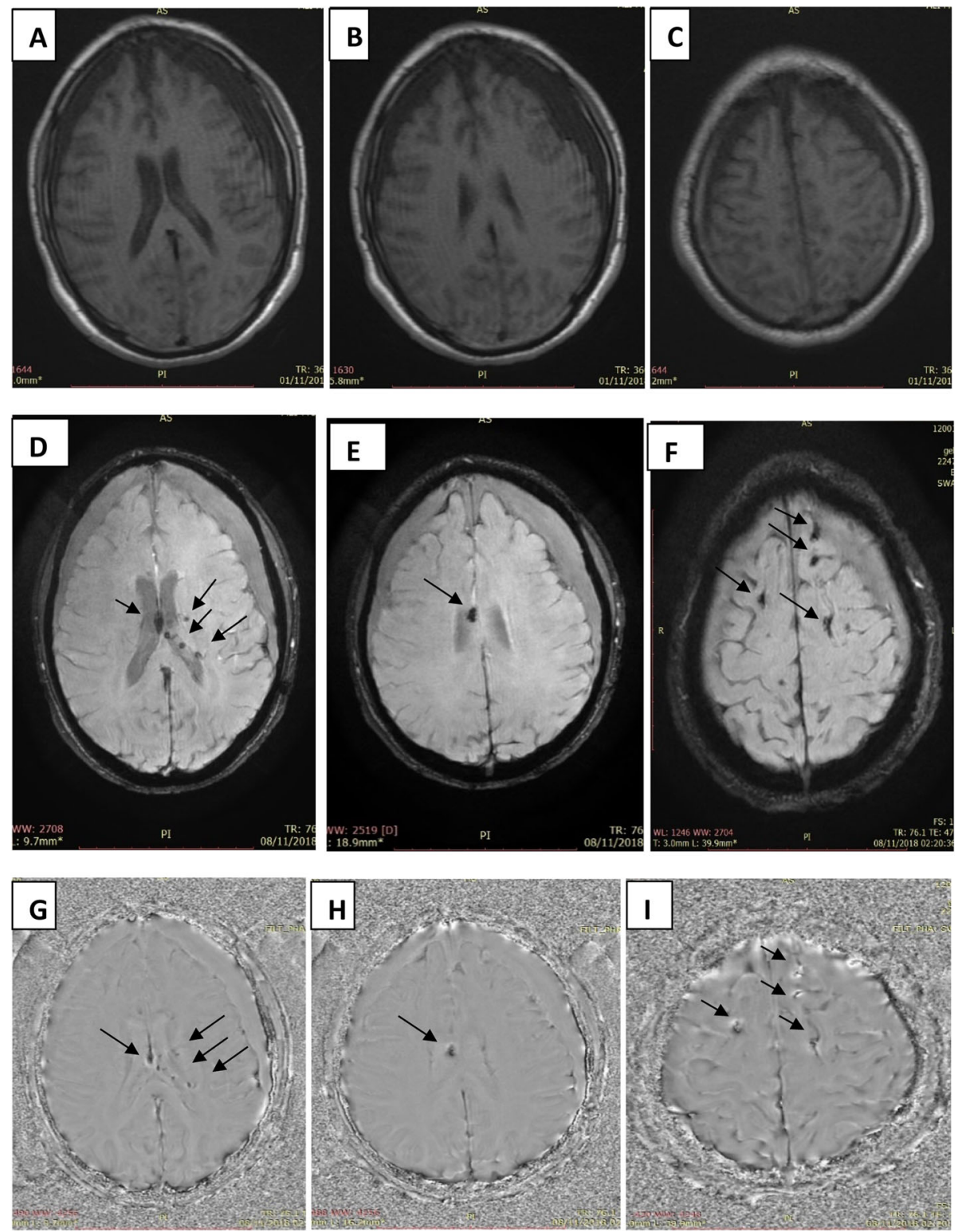

Fig. 3 Male patient aged 28 years old had road traffic accident and presented with disturbed conscious level and Glasgow coma scale = 6. MRI brain showed bilateral subdural hematomas together with multiple microhemorrhage at grey white matter interface, corpus callosum, and around the lateral ventricles denoting diffuse axonal injury that were inconspicuous in T1WI (a, b, and $\mathbf{c}$ ) but more evident in SWI (d, e, and $\mathbf{f})$ and confirmed to be microhemorrhage by filter phase $(\mathbf{g}, \mathbf{h}$, and $\mathbf{i})$ displaying hypointense $\mathrm{SI}$ 
Table $4 \mathrm{~T} 1 \mathrm{WI}, \mathrm{T} 2 \mathrm{WI}$, and SWI lesion signal intensity of diffuse axonal injury (number of patients = 10 and number of lesions = 62)

\begin{tabular}{|c|c|c|c|c|c|c|}
\hline \multirow{2}{*}{$\begin{array}{l}\text { MRI } \\
\text { sequence }\end{array}$} & \multicolumn{3}{|c|}{ Number of lesions } & \multirow{2}{*}{$\begin{array}{l}\text { Sensitivity } \\
(\%)\end{array}$} & \multirow{2}{*}{$\begin{array}{l}\text { Specificity } \\
(\%)\end{array}$} & \multirow{2}{*}{$\begin{array}{l}\text { Accuracy } \\
(\%)\end{array}$} \\
\hline & Isointense & Hypointense & Hyperintense & & & \\
\hline T1WI & 26 & 0 & 36 & 58.6 & 50 & 58.1 \\
\hline $\mathrm{T} 2 \mathrm{WI}$ & 30 & 22 & 10 & 51.7 & 50 & 51.6 \\
\hline SWI & 3 & 59 & 0 & 98.3 & 100 & 98.4 \\
\hline$P$ value & \multicolumn{6}{|c|}{$0.0067^{*}$ for SWI and $0.2^{*}$ for $(T 1 W I+T 2 W I)$} \\
\hline
\end{tabular}

*Chi-square test, $P$ value $<0.05$ is considered statistically significant

sequence defined $26(89.6 \%)$ lesions as positive and 3 $(10.3 \%)$ as negative. There were $25(86.2 \%)$ true positive, 1 (3.4\%) false positive study, $2(6.8 \%)$ true negative, and $1(3.4 \%)$ false negative study. On the other hand, T1WI defined $22(75.8 \%)$ lesions as positive and $7(24.1 \%)$ as negative. There were 18 (62\%) true positive, 4 (13.7\%) false positive, $4(13.7 \%)$ true negative, and $3(10.3 \%)$ false negative studies; also, T2WI defined 21 (72.4\%) lesions as positive and $8(27.5 \%)$ as negative. There were 16 (55.1\%) true positive, $5(17.2 \%)$ false positive, $6(20.6 \%)$ true negative, and $2(6.8 \%)$ false negative studies with sensitivity, specificity, and accuracy as shown in Table 5.

\section{Discussion}

Cerebral cavernous malformations can be sporadic or inherited, the latter characterized by multiple lesions. SWI is more sensitive in detecting the accurate number of lesions in inherited type of cerebral cavernous malformations than other sequences. In our study, 13 patients diagnosed with cavernoma, 9 of them have the inherited type with multiple lesions, and 4 have sporadic type with total number of lesions in both types $=33$. SWI could detect 30 lesions while T1WI could detect 14 lesions, and T2WI detected 21 lesions with SWI sensitivity $100 \%$, specificity $60 \%$, accuracy $93.9 \%$, and $P$ value 0.004 which was matched with Tan et al. [10] who examined 27 patients with total number of cavernomas 41 lesions stating that SWI was the highest sequence for detection of cavernomas (36 lesions, $P$ value $=0.0023$, and sensitivity 98\%). Similarly, Sparacia et al. [11] made his research on 42 patients with inherited type CCMs with total number of lesions 57 stating that 51 lesions were detectable by SWI ( $P$ value 0.0095 and sensitivity $96.5 \%$ ).

Also, because the incidence of hemorrhage from occult cerebrovascular malformations depends on presence of any previous or old microbleeds or not, so using SWI to detect microbleeds helps to predict any future risks [12].

In regard to calcification, the current study revealed 14 patients diagnosed with different pathologies including calcification with total number of lesions equals 14 interpreted with SWI and other routine MRI sequences and showed that SWI could detect 12 out of 14 lesions (85.7\%). T1WI could detect 7 out of 14 lesions $(50 \%)$, and T2WI could detect 9 out of 14 lesions (64.2) with $P$ value of SWI in detection of calcification 0.032 compared with T1WI and 0.078 compared with T2WI (that made SWI statistically insignificant in detection of calcification compared with T2WI) with sensitivity $91.7 \%$, specificity $50 \%$, and accuracy $85.7 \%$, and this matched with Malhotra et al. [13] who examined 50 patients with 76 focal calcifications and showed that SWI could detect 69 out of 76 lesions (90.7\%). T1WI could detect 40 out of 76 lesions $(52.6 \%)$, and T2WI could detect 53 out of 76 lesions (69.7\%) with $P$ value of SWI compared with T1WI that was 0.01 and mismatched with $P$ value of SWI compared with T2WI that was 0.04 , and this might be due to increased total number of patients Malhotra examined in his study.

The results of the current study were better than the study conducted by Berberat et al. [14] in which the sensitivity for detection of calcification by magnitude SWI was $86 \%$, however, and in a study conducted by Wu et al. [15], the detection rate of calcification by magnitude SWI was better than our study with detection rate $90.4 \%$, and also the location and size of calcifications perfectly matched with that of CT.

In regard to number of lesions that could be detected in DAI patients, SWI was the highest sequence in detection of the accurate number, size, and site of the lesions; as in the current study, the total number of lesions were 62 , and SWI could detect 59 lesions (95.2\%) with $P$ value $=0.0067$, sensitivity $=98.3 \%$, specificity $=100 \%$, and accuracy $=$ 98.4\%, which was in close relation to Sinha et al. [16] who showed SWI detection rate $=98.4 \%$ with $P$ value $=0.001$, sensitivity $=95 \%$, specificity $=98.5 \%$, and accuracy $=96 \%$; also, Babikian et al. [17] demonstrated that SWI is 3-6 times more sensitive than T2 $\times$ GE sequences in terms of detecting the number, size, volume, and distribution of hemorrhagic lesions seen in DAI cases. Similarly, Hamdeh et al. [18] emphasized the role of SWI in detecting lesions showing that it was better than conventional MRI sequences and also more helpful in predicting the outcome.

In our study, there were 16 patients presented with hemorrhagic lesions; some of them had multiple lesions, so the total number of lesions was 29 . SWI could detect 26 lesions $(89.6 \%)$ with $P$ value 0.028 , sensitivity $96.1 \%$, specificity $66.7 \%$, and accuracy $93.1 \%$, and this in close relation to Lawrence et al. [19]. This study encountered 

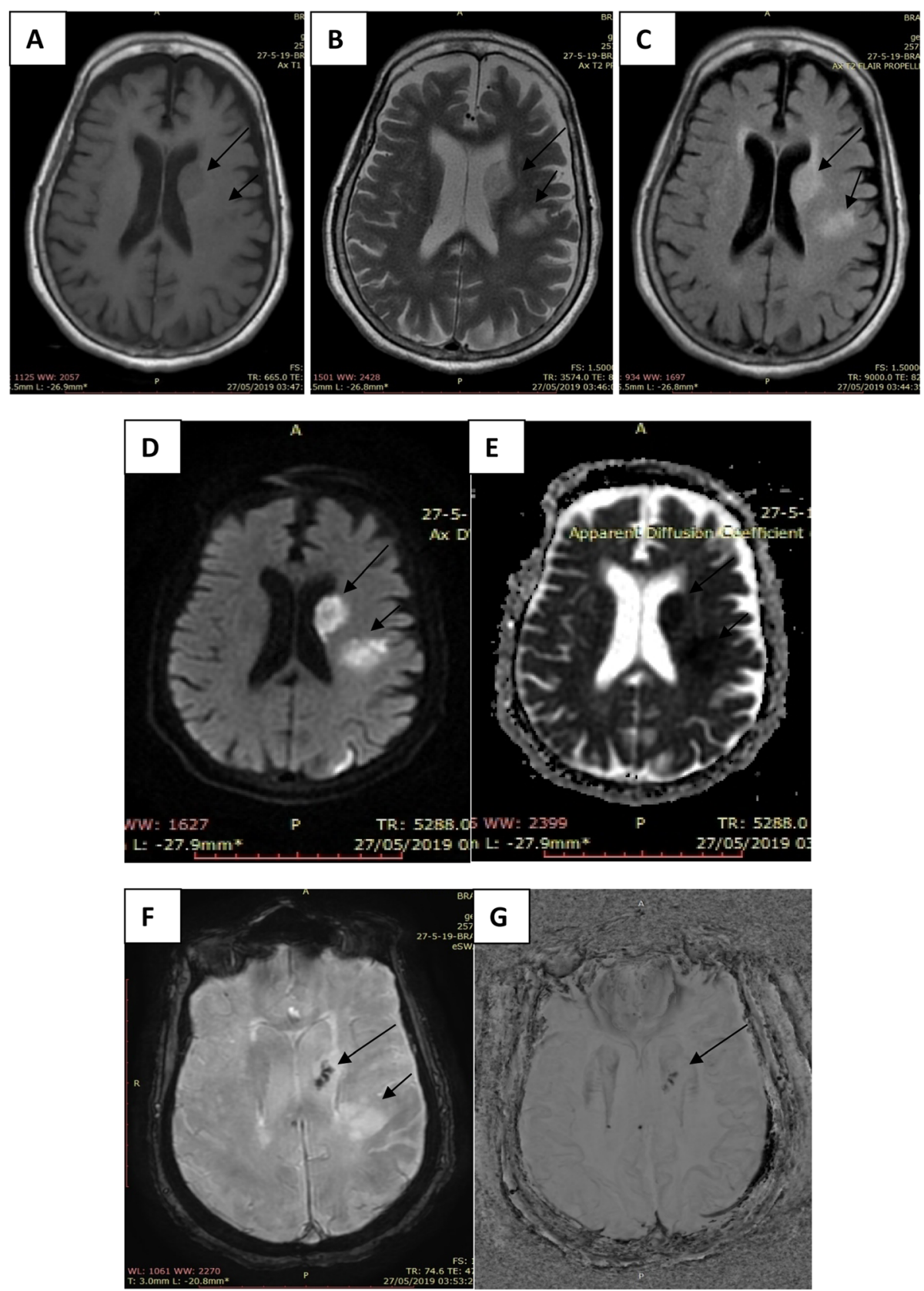

Fig. 4 Female patient aged 64 years old presented with hemiparesis and difficulty in speech. MRI brain showed left parietal acute infarction (short arrow) showing low signal intensity in T1WI (a) and high signal intensity in T2WI and FLAIR sequences (b, c) with restricted diffusion, hyperintense SI in diffusion (d), and hypointense SI in ADC (e), and no blooming effect in SWI (f) denoting non-hemorrhagic infarction together with another left basal ganglion (body of caudate) (long arrow) acute infarction with small dots of microhemorrhage seen inside by SWI (f) confirmed by low signal intensity in filter phase (g) denoting hemorrhagic infarction that was overlooked by T1WI

13 patients with total lesion number 30 and SWI detection rate $92 \%$ with $P$ value 0.03 and sensitivity $91 \%$.

In regard to the detection of chronic cerebral microbleeds, the current study revealed that 2 patients with chronic microbleeds; SWI could detect the largest number of lesions by 10 lesions while T1WI detected only 4 out of 10 lesions, and T2WI detected 6 out of 10 lesions, so SWI confers greater reliability as well as greater sensitivity for cerebral microbleed detection compared with other MRI sequences, and this matched with Cheng et al. [20] who made a study on 38 patients with chronic microbleeds and proved that SWI should be the 

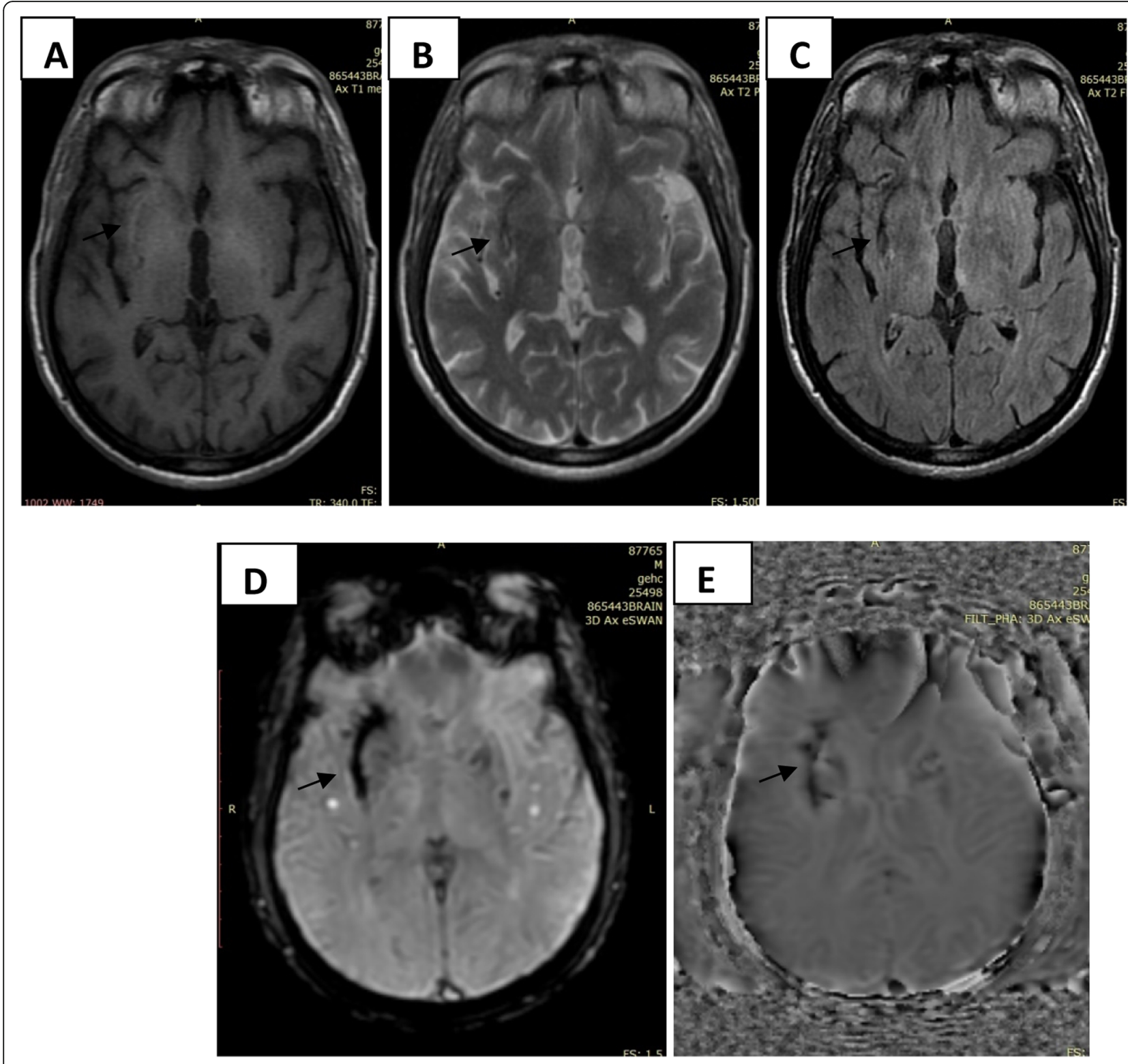

Fig. 5 Male patient aged 57 years old, presented with seizures and medical history of hypertension. MRI brain was done and showed faint

hyperintense curvillenear lesion seen in the right external capsule in T1WI (a) and displayed hypointense signal in T2WI and FLAIR sequences (b, c). SWI showed hypointense signal with blooming effect (d), and filter phase showed hypointense signal (e) denoting right external capsule hematoma

Table $5 \mathrm{~T} 1 \mathrm{WI}, \mathrm{T} 2 \mathrm{WI}$, and SWI lesion signal intensity of the hemorrhage (number of patients $=16$ and number of lesions $=29$ )

\begin{tabular}{|c|c|c|c|c|c|c|}
\hline \multirow{2}{*}{$\begin{array}{l}\text { MRI } \\
\text { sequence }\end{array}$} & \multicolumn{3}{|c|}{ Number of lesions } & \multirow{2}{*}{$\begin{array}{l}\text { Sensitivity } \\
(\%)\end{array}$} & \multirow{2}{*}{$\begin{array}{l}\text { Specificity } \\
(\%)\end{array}$} & \multirow{2}{*}{$\begin{array}{l}\text { Accuracy } \\
(\%)\end{array}$} \\
\hline & Isointense & Hypointense & Hyperintense & & & \\
\hline T1WI & 7 & 5 & 17 & 85.7 & 50 & 75.9 \\
\hline $\mathrm{T} 2 \mathrm{WI}$ & 8 & 9 & 12 & 88.9 & 54.5 & 75.9 \\
\hline SWI & 3 & 26 & 0 & 96.1 & 66.7 & 93.1 \\
\hline$P$ value & \multicolumn{6}{|c|}{$0.028^{*}$ for SWI and $0.094^{*}$ for $(\mathrm{T} 1 \mathrm{WI}+\mathrm{T} 2 \mathrm{WI})$} \\
\hline
\end{tabular}

"Chi-square test, $P$ value $<0.05$ is considered statistically significant 
preferred sequence for quantifying cerebral microbleed counts as it detected the largest number of lesions by 136 lesions.

Detection of cerebral microbleeds especially that associated with stroke patients appeared to be important biomarkers for risk of intracranial hemorrhage in case of thrombolytic therapy as presence of more than 5 microbleeds in such patients carry high risk for hemorrhagic transformation as proven by multiple studies like Halefoglu et al. [21], Chia et al. [22], and Chris et al. [23].

\section{Limitation of the study}

Our study shows some limitation. Firstly, all the patients were examined by 1.5 tesla MRI machine; further studies with $3 \mathrm{~T}$ MRI machine are recommended for better results. Secondly, the relatively long time of SWI sequence which was obstacle in few patients. Another drawback of this study was the inability to follow some patients after treatment; also, lack of CT study in few patients with calcified lesions decrease our ability to confirm the diagnosis of calcification.

\section{Conclusion}

SWI is very sensitive in the diagnosis and detection of actual number of microbleed and hemorrhage in any pathological condition like infarction, diffuse axonal injury, and vascular malformation like cavernoma and arteriovenous malformation; also, calcium with its different compounds are very susceptible substances that make SWI also very sensitive in diagnosis of calcification in various pathological lesions that were difficult to be diagnosed with conventional MRI sequences.

We recommend adding the SWI to the routine MRI in patients suspecting to have hemorrhage, vascular malformation, or calcified lesions.

\section{Abbreviations}

SWI: Susceptibility weighted imaging; FOV: Field of view; GRE: Gradient echo; TE: Echo time; TR: Repetition time; CVM: Cerebrovascular malformation; CCM: Cerebral cavernous malformation; DAl: Diffuse axonal injury; ADC: Apparent diffusion coefficient

\section{Acknowledgements}

To all the participants for their cooperation and patience.

\section{Authors' contributions}

WY suggested the research idea, ensured the original figures and data in the work, minimized the obstacles to the team of work, correlated the study concept and design, and had the major role in analysis. RE supervised the study with significant contribution to design the methodology, manuscript revision, and preparation. HA correlated the clinical data of patient and matched it with the findings, and drafted and revised the work. TG collected data in all stages of manuscript and performed data analysis. All authors read and approved the final manuscript for submission.

\section{Funding}

No funding. Not applicable for this section.

\section{Availability of data and materials}

The author's confirm that all data supporting the finding of the study are available within the article, and the raw data and data supporting the findings were generated and available at the corresponding author on request.

\section{Ethics approval and consent to participate}

Informed written consents taken from the patients and healthy volunteers, the study was approved by ethical committee of Tanta university hospital, faculty of medicine (32271/04/18).

\section{Consent for publication}

All participants included in the research gave written consent to publish the data included in the study. Authors accepted to publish the paper.

\section{Competing interests}

The authors declare that they have no competing of interests.

Received: 12 March 2020 Accepted: 5 May 2020

Published online: 28 May 2020

\section{References}

1. Hewitt J, Walters M, Dawson J et al (2016) Cohort profile of UK Biobank: diagnosis and characteristics of cerebrovascular disease. BMJ Open 6(3):4-7

2. Timothy $L$, Adivta B, Walter M et al (2015) Magnetic resonance susceptibility weighted imaging in neurosurgery: current application and future perspectives. J Neurosurg 123(6):1463-1475

3. Chunlei L, Wei L, Samuel K et al (2015) Susceptibility-weighted imaging and quantitative susceptibility mapping in the brain. J Magn Reson Imaging 42(1):23-41

4. Hayashida $Y$, Kakeda S, Hiai $Y$ et al (2014) Diagnosis of intracranial hemorrhagic lesions: comparison between 3D-SWAN (3D T2*-weighted imaging with multi-echo acquisition) and 2D-T2*-weighted imaging. Acta Radiol Stockh Swed 1987 55(2):201-207

5. Zhao G, Sun L, Yang D et al (2017) Evaluation of the role of susceptibilityweighted imaging in thrombolytic therapy for acute ischemic stroke. J Clin Neurosci 40(9):175-179

6. Hapugoda S, Craigie M, Hsu CC et al (2017) Susceptibility weighted imaging in acute cerebral ischemia: review of emerging technical concepts and clinical application. Neuroradiol J 30(2):109-119

7. Bosemani T, Verschuuren SI, Poretti A et al (2014) Pitfalls in susceptibilityweighted imaging of the pediatric brain. J Neuroimaging 24(3):221-225

8. Jun L, Robin H, Shuang $X$ et al (2016) Susceptibility weighted imaging and mapping of micro-hemorrhages and major deep veins after traumatic brain injury. J Neurotrauma 33(1):10-21

9. Song L, Lijuan Y, Lijin W (2015) Comparison of susceptibility weighted and perfusion-weighted magnetic resonance imaging in the detection of penumbra in acute ischemic stroke. J Neuroradiol 42(5):255-260

10. Tan H, Liu T, Wu Y et al (2014) Evaluation of iron content in human cerebral cavernous malformation using quantitative susceptibility mapping. Investig Radiol 49(7):498-504

11. Sparacia G, Speciale C, Banco A et al (2016) Accuracy of SWI sequence compared to $\mathrm{T}^{*}$ weighted gradient echo sequences in the detection of cerebral cavernous malformations in the familial form. J Neuroradiol 29(5): 325-336

12. Steven M, Meike W, Charlotte C et al (2009) Cerebral microbleeds: a guide to detection and interpretation. Lancet Neurol 8(2):165-174

13. Malhotra A, Azad R, Mittal P (2017) Detection and differentiation of focal intracranial calcifications and chronic microbleeds using MRI. J Clin Diagn Res 11(5):19-23

14. Berberat J, Grobholz R, Boxheimer L et al (2014) Differentiation between calcification and hemorrhage in brain tumors using susceptibility weighted imaging: a pilot study. Am J Roentgenol 202(8):847-850

15. Wu Z, Mittal S, Kish K et al (2013) Identification of calcification with MRI using susceptibility-weighted imaging: a case study. J Magn Reson Imaging 29(1):177-182

16. Sinha V, Purohit D, Karthikeyan $Y$ (2017) Role of magnetic resonance imaging in unconscious patients due to diffuse axonal injury and its prognostic value. Ind J Neurotrauma 32(7):312-344

17. Babikian T, Merkley T, Ronald C (2015) Chronic aspects of traumatic brain injury: review of Literature. J Neurotrauma 147(12):235-277 
18. Hamdeh A, Marklund N, Lannsjö M et al (2017) Extended anatomical grading in diffuse axonal injury using MRl: hemorrhagic lesions in the substantia nigra and mesencephalic tegmentum indicate poor long term outcome. J Neurotrauma 66(3):37-45

19. Lawrence T, Pretorius M, Ezra M et al (2017) Early detection of cerebral microbleeds following traumatic brain injury using MRI in the hyper-acute phase. Neurosci Lett 655(7):143-150

20. Cheng A, Batool S, McCreary R et al (2013) Susceptibility weighted imaging is more reliable than $2^{*}$ - weighted gradient-recalled echo MRI for detecting microbleeds. Stroke 44(10):13-27

21. Halefoglu M, Yousem D (2018) Susceptibility weighted imaging: clinical applications and future directions. World J Radiol 10(4):30-45

22. Chia C, Hsu T, Kwa N et al (2017) Susceptibility weighted imaging in acute cerebral ischemia: review of emerging technical concepts and clinical application. Neuroradiol J 41(8):287-290

23. Chris H, Leon A, Bharatha P et al (2016) Susceptibility-weighted imaging in neurovascular disease. Top Magn Reson Imaging 25(2):63-71

\section{Publisher's Note}

Springer Nature remains neutral with regard to jurisdictional claims in published maps and institutional affiliations.

\section{Submit your manuscript to a SpringerOpen ${ }^{\circ}$ journal and benefit from:}

- Convenient online submission

- Rigorous peer review

- Open access: articles freely available online

High visibility within the field

- Retaining the copyright to your article

Submit your next manuscript at $\boldsymbol{\nabla}$ springeropen.com 